\title{
"Fan-zhen Jie-ci" acupuncture therapy for treatment of discogenic sciatica: protocol for a single-blind, randomized controlled clinical trial
}

\author{
Wen-Guang Hou ${ }^{1 \#}$, Hao-Tian Pan ${ }^{2 \#}$, Rui-Ping Wang ${ }^{3}$, Chen-Chen Feng ${ }^{2}$, Li-Juan Pei ${ }^{2}$, Jing Li ${ }^{1}$, \\ Jia Zhou ${ }^{2}$, Ke Wang ${ }^{2} \wedge$ \\ ${ }^{1}$ Department of Acupuncture-moxibustion, Yueyang Hospital of Integrated Traditional Chinese and Western Medicine, Shanghai University of \\ Traditional Chinese Medicine, Shanghai, China; ${ }^{2}$ Acupuncture Anesthesia Clinical Research Institute, Yueyang Hospital of Integrated Traditional Chinese \\ and Western Medicine, Shanghai University of Traditional Chinese Medicine, Shanghai, China; ${ }^{3}$ Office of National Clinical Research Base of TCM, \\ Yueyang Hospital of Integrated Traditional Chinese and Western Medicine, Shanghai University of Traditional Chinese Medicine, Shanghai, China \\ Contributions: (I) Conception and design: K Wang, J Zhou, WG Hou; (II) Administrative support: K Wang, J Zhou; (III) Provision of study materials \\ or patients: WG Hou, HT Pan, J Li; (IV) Collection and assembly of data: CC Feng, LJ Pei; (V) Data analysis and interpretation: RP Wang, K \\ Wang, J Zhou; (VI) Manuscript writing: All authors; (VII) Final approval of manuscript: All authors. \\ "These authors contributed equally to this work. \\ Correspondence to: Ke Wang; Jia Zhou. Acupuncture Anesthesia Clinical Research Institute, Yueyang Hospital of Integrated Traditional Chinese \\ and Western Medicine, Shanghai University of Traditional Chinese Medicine, No. 110 Ganhe Road, Hongkou District, Shanghai 200437, China. \\ Email: wangke8430@163.com; pdzhoujia@163.com.
}

Background: Curative medical treatment for patients with discogenic sciatica is limited. Acupuncture
is an important non-pharmacological therapy reported to have positive therapeutic effects on discogenic
sciatica. According to traditional Chinese acupuncture theory, discogenic sciatica is a channel tendon disease
which can be treated by a special "Fan-zhen Jie-ci (FZJC)" method. Our recent preliminary clinical evidence
indicated that the FZJC method had a positive treatment effect on the disease. This study will further
evaluate the efficacy and safety of FZJC on patients with discogenic sciatica.

Methods: A single-blind randomized controlled clinical trial will be conducted, assigning 76 participants with discogenic sciatica to a randomly assigned FZJC group or a control group. Acupuncture treatment combined with the FZJC method will be applied in the FZJC group while routine acupuncture treatment only will be applied in the control group. Treatments will be administered three times a week for a total of 3 consecutive weeks. The primary outcome of this trial is change in the visual analogue scale of leg pain (VASLP), and the secondary outcomes are changes in the visual analogue scale of back pain (VAS-BP), the Brief Pain Inventory (BPI), the Oswestry Disability Index (ODI), the 36-Item Short Form Health Survey (SF-36), and serum concentrations of mitochondrial DNA (mtDNA) and high sensitivity C reactive protein (hsCRP).

Discussion: The results of this study will provide insight into the efficacy and safety of FZJC acupuncture as a treatment for discogenic sciatica.

Trial registration: The trial has been registered at the Chinese Clinical Trial Registry (ChiCTR1900026272) and the Acupuncture-Moxibustion Clinical Trial Registry (AMCTR-IOR-19000275).

Keywords: Acupuncture; Fan-zhen Jie-ci acupuncture therapy; randomized controlled trial (RCT); sciatica; traditional Chinese medicine (TCM)

Submitted Jul 16, 2020. Accepted for publication Nov 04, 2020.

doi: 10.21037/apm-20-1418

View this article at: http://dx.doi.org/10.21037/apm-20-1418

$\wedge$ ORCID: 0000-0002-5845-7228. 


\section{Introduction}

Sciatica is characterized by a radiating posterior or posterolateral leg pain accompanied by sensory and motor dysfunction and caused by compression of the lumbosacral nerve roots resulting in inflammation $(1,2)$. The prevalence of sciatica is estimated to be within the range of $1.2 \%$ to $43 \%$ and approximately $85 \%$ of sciatica patients suffer from discogenic sciatica $(3,4)$. The current treatment methods are primarily surgical and conservative therapies (5). Surgery is an effective treatment method that provides faster pain relief to patients with severe sciatica, but there is no significant difference in clinical outcomes between surgery and conservative treatment during the first 4 years after surgery (6-8). Conservative therapy is generally recommended to manage pain and maintain function in the absence of a major neurologic deficit (8). Although pharmacological treatments are widely used, evidence regarding their curative effect is limited and their use can lead to adverse effects $(5,9)$. Recently, the American College of Physicians (ACP) has recommended non-pharmacological therapies, including exercise, spinal mobilization, massage, and acupuncture, for the treatment of sciatica (9).

Acupuncture has been widely used for pain control across the world and a 10-year self-comparison study reported that this treatment provides satisfactory longterm clinical effects for lumbar disc herniation and sciatica $(10,11)$. Although evidence is limited, systematic reviews attest to the benefits of acupuncture for the treatment of sciatica $(12,13)$. Recent randomized controlled trials (RCTs) revealed that acupuncture had short-term clinical benefits for chronic discogenic sciatica compared with sham acupuncture and had greater short-term and long-term benefits in comparison to medium-frequency electrotherapy $(14,15)$.

Channel tendon theory and channel tendon disease are important components of acupuncture treatment, and their history can be dated to the Miraculous Pivot, an important part of the Huangdi Internal Classic. As the first classic monograph on acupuncture, the Miraculous Pivot records "Fan-zhen Jie-ci (FZJC)" as a special acupuncture method to treat channel tendon disease. "Fan-zhen" means using warm needle or fire needle to stimulate the specific acupoint, which yields both ordinary acupuncture and warming effects. A series of studies have shown that this method is superior to ordinary needle stimulation for the treatment of various diseases, such as chronic non-bacterial prostatitis, post-stroke shoulder-hand syndrome, and third lumbar vertebrae syndrome (16-18). "Jie-ci", meanwhile, refers to inserting the needle directly into the pain point and then withdrawing it quickly, which is believed to quickly remove pathogenic evils. A randomized controlled study using fast acupuncture for patients with cervical spondylosis showed that this treatment was superior to routine retaining acupuncture (19). Fire needle therapy is a representative of the FZJC method in the treatment of disease, and consists of a red-hot needle piercing into an acupoint and then quickly being removed. Some clinical reports have shown that fire needle therapy is an effective treatment for pain $(20,21)$. According to the channel tendon theory, channel tendon diseases are typically characterized by pain and movement disorders, involving several locations, including the neck, shoulder, lumbar region, and leg (22). Moreover, the hallmark of sciatica is that pain is felt in the sciatic-nerve distribution, which is consistent with the main distribution of the channel tendon of Foot-taiyang and Foot-shaoyang. On this basis, sciatica is considered a typical type of channel tendon disease of Foot-taiyang and Foot-shaoyang $(22,23)$, and the FZJC method is used to treat it in our clinical practice. Based on our clinical observations, we found that the FZJC method effectively relieves pain in patients with discogenic sciatica. To our knowledge, no comparative studies on the efficacy and safety of FZJC and routine acupuncture treatment using RCTs have been conducted. We designed this RCT to assess FZJC efficacy and safety in managing discogenic sciatica and present the following article in accordance with the SPIRIT reporting checklist (available at http://dx.doi.org/10.21037/apm-20-1418).

\section{Methods and design}

\section{Experimental design}

A single-center, double-blind (patient and assessor), randomized controlled trial will be conducted. The aim is to assess the clinical efficacy and safety of FZJC relative to routine acupuncture treatment in managing discogenic sciatica. The findings of the study may be utilized in promoting the clinical applications of FZJC acupuncture technique. The study protocol (version: 20190415-2.0) has been approved by the Ethics Committee of the Yueyang Hospital of Integrated Traditional Chinese and Western Medicine, Shanghai University of Traditional Chinese Medicine (approval number: 2019-036). All study participants provide their informed consent before inclusion into the study. The trial is registered at the Chinese Clinical 


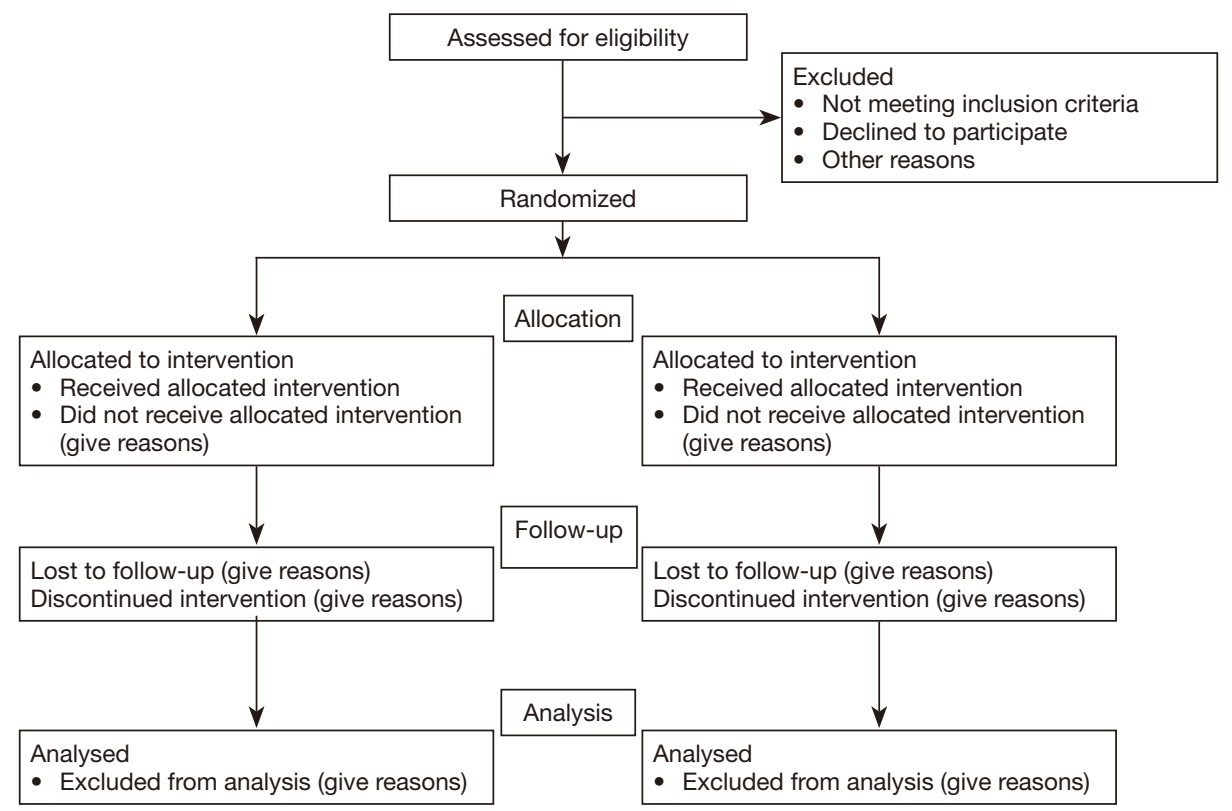

Figure 1 Flow diagram through the study. FZJC, Fan-zhen Jie-ci.

Trial Registry (http://www.chictr.org.cn/abouten.aspx; no: ChiCTR1900026272) and the Acupuncture-Moxibustion Clinical Trial Registry (http://www.acmctr.org/; no: AMCTR-IOR-19000275). The study protocol is in line with the Standards for Reporting Interventions in Clinical Trials for Acupuncture (STRICTA), Consolidated Standards of Reporting Trials (CONSORT), and the Standard Protocol Items: Recommendations for Interventional Trials (SPIRIT) $2013(24,25)$. If there are any changes in study design, the Ethics Committee will be immediately informed. The study will be performed at the Yueyang Hospital of Integrated Traditional Chinese and Western Medicine, Shanghai University of Traditional Chinese Medicine. Seventy-six patients with discogenic sciatica will be included and randomly assigned at a ratio of $1: 1$ to the FZJC or control group after acquisition of informed consent and confirmation of eligibility. During enrollment, participants recruited from the acupuncture-moxibustion clinic setting will undergo physical examination and eligibility assessment and a trained medical staff member will extensively explain the details of the trial. A study investigator will communicate with the participants 1 day in advance of trial commencement via phone and WeChat to remind them of their visit. Figure 1 shows the participant flowchart, and Table 1 presents the schedule of enrollment, interventions, and assessments.

\section{Participation and recruitment}

\section{Inclusion criteria}

The following inclusion criteria for patients will be used in the study: (I) between 18 and 75 years of age; (II) have experience of unilateral leg pain diagnosed as discogenic sciatica (ICD-10 codes: M54.381), and leg pains that coincide with the results of computed tomography (CT) or magnetic resonance imaging (MRI) of lumbar disc herniation; (III) have experience of an average leg pain visual analogue scale (VAS-LP) of $40 \mathrm{~mm}$ in the previous 24 hours; (IV) agreement to follow the trial protocol and voluntarily sign an informed consent form.

\section{Exclusion criteria}

The following exclusion criteria for patients will be used in the study: (I) severe cases of ruptured, central, or giant disc herniation, or foot drop, cauda equina syndrome, or need for surgery; (II) neurological symptoms that have progressed after 3 months of strict conservative treatment, which include nerve root adhesion, obvious muscle atrophy, or crossed straight-leg testing; (III) severe autoimmune, cardiovascular, liver, hematopoietic system, or kidney disease, or poor nutritional status; (IV) cognitive impairment; (V) pregnant women (positive human chorionic gonadotropin test); (VI) acupuncture for sciatica applied within the previous month. 
Table 1 Schedule of enrolment, interventions and assessments

\begin{tabular}{|c|c|c|c|c|c|}
\hline Activity & Phase (time points) & $\begin{array}{c}\text { Enrolment } \\
\text { (Week 0) }\end{array}$ & \multicolumn{3}{|c|}{ Treatment/intervention } \\
\hline \multirow[t]{3}{*}{ Enrolment } & Eligibility screen & $\bullet$ & & & \\
\hline & Informed consent & $\bullet$ & & & \\
\hline & Allocation & $\bullet$ & & & \\
\hline Interventions & FZJC acupuncture treatment ${ }^{\#}$ & & $\bullet$ & $\bullet$ & $\bullet$ \\
\hline \multirow[t]{5}{*}{ Outcome assessment } & VAS-LP & $\bullet$ & $\bullet$ & $\bullet$ & $\bullet$ \\
\hline & VAS-BP & $\bullet$ & & & $\bullet$ \\
\hline & $\mathrm{BPI}$ & $\bullet$ & & & $\bullet$ \\
\hline & Serum hsCRP & $\bullet$ & & & $\bullet$ \\
\hline & Serum mtDNA & $\bullet$ & & & $\bullet$ \\
\hline Safety assessment & AEs & $\bullet$ & $\bullet$ & $\bullet$ & $\bullet$ \\
\hline
\end{tabular}

\section{Stopping criteria}

If the principal investigator asserts that there are unacceptable risks for serious adverse events during the study, it will be stopped.

\section{Randomization and grouping}

Upon confirmation of eligibility and receipt of informed consent, the participants will be randomly assigned into the FZJC and control groups. An independent statistician will provide computer-generated random sequences through Statistics Analysis System (SAS) software which will be ranked in order. The random sequence for group classification will be sealed in opaque envelopes and numbered consecutively according to the rank sequence.

\section{Blinding method}

Participants will be blinded to the kind of acupuncture treatment they are receiving, and the acupuncturist will be trained not to discuss the type of treatment being applied. At the end of the final treatment, the effectiveness of blinding will be assessed using the blinding index (26).
Statisticians and outcome assessors will also be blinded to the grouping of participants during the trial.

\section{Intervention method}

The acupuncture treatment protocol is based on the Clinical Practice Guideline for Low Back Pain Treated with Acupuncture and Moxibustion (27). Participants will undergo 3 treatment sessions each week for three consecutive weeks. The acupuncturists delivering treatment for both groups will be registered practitioners of Traditional Chinese Medicine (TCM) with at least 5 years of previous clinical experience. Moreover, all acupuncturists will receive special training for the standardization of the acupuncture treatment and manipulation prior to the trial. Acupoints of the affected side of Huantiao (GB30), and the bilateral Shenshu (BL23), Dachangshu (BL25), Weizhong (BL40) and lumbar Jiaji (EX-B2) will be included in both groups. In addition, Ashi points will be chosen by the acupuncturists according to the participant's lumbosacral tenderness. The Huantiao (GB30), Shenshu (BL23), Dachangshu (BL25), and Weizhong (BL40) will be located based on the World Health Organization Standardized 
Acupuncture Location. The Lumbar Jiaji (Ex-B2) is situated on both lower sides of the L1 to L5 spinous process and 0.5 inches lateral to the posterior median line (Figure S1). Disposable, sterile acupuncture needles (Hwato Needles, Sino-foreign Joint Venture Suzhou Hwato Medical Instruments, China) will be used.

In both groups, the needles $(0.25 \mathrm{~mm} \times 40 \mathrm{~mm}$ in size $)$ will be inserted straight into the Shenshu, Dachangshu, Weizhong, Jiaji, and Ashi acupoints to a depth of 15-25 mm. After insertion, the needles will be manually manipulated by lifting, thrusting, twirling and rotating to produce the sensation of "Deqi". Deqi is a sensation soreness, distention, numbness, or heaviness surrounding the acupuncture point that is felt by the participant and perceived by the acupuncturist as subcutaneous tension around the needle. Needles will be retained for 20 minutes in each acupuncture session.

The two groups will be allocated to different interventions at Jiaji and Huantiao acupoints. After the needles are inserted into Jiaji acupoints to produce the Deqi sensation, the participants of the FZJC group will undergo extra manual acupuncture therapy. A moxa stick (Nanyang bencaoai Development Co., Ltd, Nanyang, Henan, China) of $12 \mathrm{~mm}$ diameter and $15 \mathrm{~mm}$ length will be attached to each needle handle. The center of the cylindrical moxa stick will be inserted into the needle handle and placed $3 \mathrm{~cm}$ away from the skin surface, after which the bottom of the stick will be ignited to burn for approximately 10 minutes. The same procedure will be followed using a second moxa stick, and the needles will be removed after the two sticks have burned. At the Huantiao acupoint, the needle $(0.35 \mathrm{~mm} \times$ $75 \mathrm{~mm}$ in size) will be inserted into a depth of $40-60 \mathrm{~mm}$, and manually manipulated to introduce a feeling of electric shock and distention sensation, upon which the needle will be immediately removed. However, in the control group, the needle will be inserted into the Huantiao acupoint at a depth of 10-15 mm, and then slowly removed.

Clinical investigators will provide participants of either group with essential medical education information, which will include staying active and engaging in appropriate physical exercise and avoiding bed rest and negative emotions.

\section{Outcome evaluation}

\section{Primary outcome}

The primary outcome is a change in leg pain intensity based on the visual analog scale (VAS) during the 3-week treatment period. The VAS is a $10-\mathrm{cm}$ continuous line scale that ranges from 0 (no pain) to 10 (unimaginable pain) (28). Participants will be asked to describe their average leg pain intensity in the 24 hours prior to treatment. The VAS-LP will be assessed and recorded before acupuncture treatment as the baseline value and weekly during the treatment duration.

\section{Secondary outcomes}

The following secondary outcome measures will be assessed: (I) alterations in average low-back pain intensity in the previous 24 hours as determined by VAS starting from baseline to treatment endpoint. (II) Change in the Brief Pain Inventory (BPI) from baseline to treatment endpoint: the Short Form of the BPI will be used, which measures pain intensity and pain interference with life (29). (III) Change to the Oswestry Disability Index (ODI) from baseline to treatment endpoint: the ODI self-administered questionnaire consists of 10 items measuring pain-related dysfunction (30). (IV) Change in the 36-Item Short Form Health Survey (SF-36) subscale score starting from baseline to treatment endpoint: the SF-36 questionnaire measures health-related quality of life that includes eight subscales to assess eight aspects of health status and one item that reflects the perceived change in health (31). (V) Alterations in serum high sensitivity $\mathrm{C}$ reactive protein (hsCRP) level and mitochondrial DNA (mtDNA) starting from baseline to treatment endpoint: these may be sensitive indicators of pain and inflammation in the peripheral blood of patients with neuropathic pain $(32,33)$ : the association between pain, disability, quality of life in patients and the values of hsCRP and mtDNA will be assessed.

\section{Safety evaluation}

Any adverse events (AEs) related to the treatment such as forgotten or broken needles, local hematoma or pain, fainting, dizziness, vertigo, or skin allergy in the punctured region after treatment, will be recorded in detail in the case report forms (CRFs). The $\mathrm{AE}$ data will include the time of occurrence, degree of $\mathrm{AE}$, possible causes, corresponding management, and need for patient withdrawal from the clinical trial. The incidence of AEs will also be assessed and presented as the number of AEs per the number of acupuncture sessions (\%).

\section{Sample size}

This study hypothesizes that acupuncture treatment by 
FZJC can achieve a significant reduction in the VASLP value compared to routine acupuncture treatment. As such, the primary outcome of this trial will be a change in leg pain intensity from baseline to treatment endpoint as measured by the VAS. To our knowledge, this is the first clinical trial to evaluate the effect of the FZJC on VAS-LP. For this reason, we first carried out a pilot study involving 30 patients (15 in each group), in which the intervention methods, acupoints, and sessions of an FZJC and control group were consistent with the proposed study. Based on the formula for sample size calculation, and our pilot study results, the mean and standardized deviations in the change of VAS-LP are $3.82 \pm 2.14$ and $2.37 \pm 2.13$ in the FZJC group and control group, respectively.

$$
n_{1}=n_{2}=\frac{2\left(z_{\alpha / 2}+z_{\beta}\right)^{2} \times \sigma^{2}}{\left(\mu_{2}-\mu_{1}\right)^{2}}
$$

With this in mind, we assume that the mean value of VAS-LP change is 3.82 in the FZJC group $\left(\mu_{2}\right)$, and 2.37 in the control group $\left(\mu_{1}\right)$. The standardized deviation in VAS-LP change is $2.14(\sigma)$, the test power is 0.8 , and the inspection level $(\alpha)$ is set to 0.05 . Thus, $\beta=1-0.8=0.2$. The two-sided tests will require 34 participants in each group. Based on the $10 \%$ probability of loss to follow-up, the proposed clinical trial will require 76 participants allocated to two groups of 38 .

\section{Data management and monitoring}

The CRFs for every participant will be recorded by trained clinical research staff. This data will be independently fed into electronic case report forms by two researchers once participant data are collected and recorded on the original CRFs upon the completion of treatment. The consistency and accuracy of the data will then be validated and checked by another independent supervisor. All hard copy documents and corresponding electronic data will be stored separately and preserved for at least 5 years after study completion.

\section{Statistical analysis}

All outcomes will be assessed based on the principle of intention-to-treat (ITT). The missing value will be filled in using the last-observation-carried-forward method. Data will be analyzed by an independent statistician who is blinded to the group assignments. Continuous variables will be presented as the mean \pm standard deviation for data with normal distribution, where median and interquartile range (Q1, Q3) will be employed for data with a skewed distribution. Categorical variables will be expressed as frequencies (percentages). Independent two-sample $t$-tests will be employed for comparison between groups, whereas paired $t$-tests will be utilized for within-group comparisons when a normal distribution of continuous variables is observed. Generalized linear models (GLM) will be applied for repeated measures of continuous variables. The MannWhitney rank-sum test will be employed for continuous variables with skewed distribution and the Chi-square test will be used for categorical variables. The Mann-Whitney rank-sum test will also be used for non-normally distributed variables. All data in the study will be analyzed using the SPSS 19.0 statistical software package (IBM Co., Armonk, NY, USA). Two-tailed analyses will be performed, and statistical significance will be defined as a $\mathrm{P}$ value $<0.05$.

\section{Discussion}

Discogenic sciatica is a major health concern across the world and incurs an extensive individual, social, and economic burden. Conservative treatment measures serve as the first line of treatment of disc herniation patients with radicular pain (34). Systematic reviews, meta-analyses, and RCTs of treatments options suggest that acupuncture is a promising treatment strategy for sciatica (12-15).

According to TCM theory, it is very important to apply appropriate needle manipulation techniques and stimulation in the acupoint area. While there are many kinds of manipulation techniques and stimulation methods in traditional acupuncture theory, few trials have conclusively evaluated whether different acupoint stimulation and manipulation achieve the same treatment outcomes. Muscle region theory and channel tendon disease are important components of traditional acupuncture theory, and FZJC is a special manipulation technique and stimulation method specific for channel tendon disease. In this trial, the efficacy of FZJC acupuncture therapy for patients with discogenic sciatica will be evaluated based on muscle region theory and the clinical experience of medical practitioners. One meta-analysis reported that warm needle treatment is superior in efficiency rate, excellence rate, and pain control for lumbar disc herniation compared with routine acupuncture (35). Fire needle therapy will cause a microscalding reaction at the stimulation acupoint, which may easily cause infection if not managed appropriately (36). Accordingly, we will apply warm needle stimulation at 
JiaJi acupoints for the FZ method. Previous studies have suggested that inducing an electric shock feeling and distention sensation at the Huantiao acupoint is important to achieving better treatment effects $(37,38)$. The needle should be withdrawn quickly after the patient feels this sensation as this may cause sciatic nerve damage (38). Thus, for the JC portion, we will apply this method to stimulate the Huantiao acupoint. Another clinical study showed that stimulating the Huantiao acupoint in this way is more effective in relieving sciatica than routine acupuncture (39). In this clinical trial, a generally-accepted scale will be used for classifying the outcomes. Moreover, the blood samples of participants will be collected to detect serum hsCRP and mtDNA concentrations. Although hsCRP is a sensitive indicator, results from using it as a predictor of curative effect in patients with sciatica are contradictory $(40,41)$. Circulating mtDNA copy number can change in response to physiological signals and disease status (42). In recent years, circulating mtDNA content has been proposed as an emerging biomarker for several diseases or risk prediction (43-45). Some studies also show that a statistically significant positive correlation exists between mtDNA content and low-grade systemic inflammation and pain $(33,46)$. This trial will evaluate the dynamic changes of mtDNA and hsCRP concentration, and pain, disability, and quality of life in patients with discogenic sciatica during acupuncture treatment in both groups. In an innovative aspect of the study, changes in mtDNA and hsCRP concentration may also further determine the possibility of their being used as biomarkers to monitor treatment effects. This study is limited by the impracticability of doubleblinding because therapists will have to carry out the acupuncture procedures.

\section{Conclusions}

This trial will generate detailed and accurate evidence on the efficacy and safety of FZJC acupuncture for the treatment of discogenic sciatica. Furthermore, confirmation of the hypothesis may have a profound impact on muscle region theory that may contribute to future changes in the clinical practice of acupuncture therapy.

\section{Acknowledgments}

Funding: This study has been supported by Grants from the Medical Guidance Project of the Shanghai Science and Technology Commission (No. 18401904900), National
Natural Science Foundation of China (No. 81673756, 81973940), Shanghai Health System Talent Training Program (No. 2018BR24), Accelerating the Development of Chinese Medicine Three-Year Action Plan of Shanghai (No. ZY(2018-2020)-CCCX-2004-04), and Clinical Key Specialty Construction Foundation of Shanghai (No. shslczdzk04701). The authors are grateful for the financial support of these institutions.

\section{Footnote}

Reporting Checklist: The authors have completed the SPIRIT reporting checklist. Available at http://dx.doi.org/10.21037/ apm-20-1418

Conflicts of Interest: All authors have completed the ICMJE uniform disclosure form (available at http://dx.doi. org/10.21037/apm-20-1418). WGH reports grants from Shanghai Science and Technology Commission, grants from National Natural Science Foundation of China, grants from Shanghai Municipal Health Commission, grants from Shanghai Municipal Administrator of Traditional Chinese Medicine, during the conduct of the study. HTP reports grants from Shanghai Science and Technology Commission, grants from National Natural Science Foundation of China, grants from Shanghai Municipal Health Commission, grants from Shanghai Municipal Administrator of Traditional Chinese Medicine, during the conduct of the study. RPW reports grants from Shanghai Science and Technology Commission, grants from National Natural Science Foundation of China, grants from Shanghai Municipal Health Commission, grants from Shanghai Municipal Administrator of Traditional Chinese Medicine, during the conduct of the study. CCF reports grants from Shanghai Science and Technology Commission, grants from National Natural Science Foundation of China, grants from Shanghai Municipal Health Commission, grants from Shanghai Municipal Administrator of Traditional Chinese Medicine, during the conduct of the study. LJP reports grants from Shanghai Science and Technology Commission, grants from National Natural Science Foundation of China, grants from Shanghai Municipal Health Commission, grants from Shanghai Municipal Administrator of Traditional Chinese Medicine, during the conduct of the study. JL reports grants from Shanghai Science and Technology Commission, grants from National Natural Science Foundation of China, grants from Shanghai Municipal Health Commission, grants from Shanghai Municipal Administrator of Traditional Chinese 
Medicine, during the conduct of the study. JZ reports grants from Shanghai Science and Technology Commission, grants from National Natural Science Foundation of China, grants from Shanghai Municipal Health Commission, grants from Shanghai Municipal Administrator of Traditional Chinese Medicine, during the conduct of the study. KW reports grants from Shanghai Science and Technology Commission, grants from National Natural Science Foundation of China, grants from Shanghai Municipal Health Commission, grants from Shanghai Municipal Administrator of Traditional Chinese Medicine, during the conduct of the study. The authors have no other conflicts of interest to declare.

Ethical Statement: The authors are accountable for all aspects of the work in ensuring that questions related to the accuracy or integrity of any part of the work are appropriately investigated and resolved. This trial protocol has been approved by the Ethics Committee of Yueyang Hospital of Integrated Traditional Chinese and Western Medicine, Shanghai University of Traditional Chinese Medicine (No. 2019-036). Informed consent is obtained from each patient.

Open Access Statement: This is an Open Access article distributed in accordance with the Creative Commons Attribution-NonCommercial-NoDerivs 4.0 International License (CC BY-NC-ND 4.0), which permits the noncommercial replication and distribution of the article with the strict proviso that no changes or edits are made and the original work is properly cited (including links to both the formal publication through the relevant DOI and the license). See: https://creativecommons.org/licenses/by-nc-nd/4.0/.

\section{References}

1. Ropper AH, Zafonte RD. Sciatica. N Engl J Med 2015;372:1240-8.

2. Valat JP, Genevay S, Marty M, et al. Sciatica. Best Pract Res Clin Rheumatol 2010;24:241-52.

3. Konstantinou K, Dunn KM. Sciatica: review of epidemiological studies and prevalence estimates. Spine (Phila Pa 1976) 2008;33:2464-72.

4. Porchet F, Wietlisbach V, Burnand B, et al. Relationship between severity of lumbar disc disease and disability scores in sciatica patients. Neurosurgery 2002;50:1253-9.

5. Jensen RK, Kongsted A, Kjaer P, et al. Diagnosis and treatment of sciatica. BMJ 2019;367:16273.
6. Weinstein JN, Lurie JD, Tosteson TD, et al. Surgical vs nonoperative treatment for lumbar disk herniation: the Spine Patient Outcomes Research Trial (SPORT) observational cohort. JAMA 2006;296:2451-9.

7. Peul WC, van Houwelingen HC, van den Hout WB, et al. Surgery versus prolonged conservative treatment for sciatica. N Engl J Med 2007;356:2245-56.

8. Deyo RA, Mirza SK. Clinical practice. Herniated Lumbar Intervertebral Disk. N Engl J Med 2016;374:1763-72.

9. Qaseem A, Wilt TJ, McLean RM, et al. Noninvasive Treatments for Acute, Subacute, and Chronic Low Back Pain: A Clinical Practice Guideline From the American College of Physicians. Ann Intern Med 2017;166:514-30.

10. NIH Consensus Conference. Acupuncture. JAMA 1998;280:1518-24.

11. Gu H. A 10-year follow-up of electric acupuncture for lumbar disc herniation and sciatica. World J Acupunct Moxibustion 2019;29:221-3.

12. Qin Z, Liu X, Wu J, et al. Effectiveness of Acupuncture for Treating Sciatica: A Systematic Review and Meta-Analysis. Evid Based Complement Alternat Med 2015;2015:425108.

13. Ji M, Wang X, Chen M, et al. The Efficacy of Acupuncture for the Treatment of Sciatica: A Systematic Review and Meta-Analysis. Evid Based Complement Alternat Med 2015;2015:192808.

14. Huang Z, Liu S, Zhou J, et al. Efficacy and Safety of Acupuncture for Chronic Discogenic Sciatica, a Randomized Controlled Sham Acupuncture Trial. Pain Med 2019;20:2303-10.

15. Zhang $X$, Wang $Y$, Wang Z, et al. A Randomized Clinical Trial Comparing the Effectiveness of Electroacupuncture versus Medium-Frequency Electrotherapy for Discogenic Sciatica. Evid Based Complement Alternat Med 2017;2017:9502718.

16. Chen ZX. Observation on therapeutic effect of warm needle moxibustion on chronic non-bacterial prostatitis. Zhongguo Zhen Jiu 2009;29:275-8.

17. Meng FY, Wen J. Effect of warm acupuncture stimulation of Waiguan (TE 5) on post-stroke shoulder-hand syndrome. Zhen Ci Yan Jiu 2014;39:228-31.

18. Xu F. Needle warming therapy for the treatment of syndrome of the transverse process of the third lumbar vertebra. Zhongguo Gu Shang 2010;23:440-3.

19. Li W, Cong W, Yan C, et al. Clinical observation of fast acupuncture for cervical type of cervical spondylosis. Zhongguo Zhen Jiu 2017;37:951-4.

20. Yang LY, Lu DJ, Li YH. Observation on therapeutic effect of fire-needle therapy on lumbar intervertebral disc 
herniation. Zhongguo Zhen Jiu 2009;29:449-51.

21. Wang JX, Zhao WX, Zeng JC, et al. Systematic review and sequential analysis on treatment of herpes zoster pain mainly by fire needle therapy. Zhen Ci Yan Jiu 2019;44:677-85.

22. Liu RB, Kong SY, Yao N, et al. Clinical Research Status of Treatment of Low Back Pain based on Meridian Tendons Theory. Zhongguo Min Zu Min Jian Yi Yao 2019;28:41-3.

23. Wei YC, Zhu HM. Treating Sciatica from channel tendon theory. Gan Su Zhong Yi 1993;6:6-7.

24. MacPherson H, Altman DG, Hammerschlag R, et al. Revised STandards for Reporting Interventions in Clinical Trials of Acupuncture (STRICTA): extending the CONSORT statement. J Altern Complement Med 2010;16:ST1-14.

25. Chan AW, Tetzlaff JM, Altman DG, et al. SPIRIT 2013 statement: defining standard protocol items for clinical trials. Ann Intern Med 2013;158:200-7.

26. Kolahi J, Bang H, Park J. Towards a proposal for assessment of blinding success in clinical trials: up-to-date review. Community Dent Oral Epidemiol 2009;37:477-84.

27. Zhao H, Liu Z, Xie L, et al. Interpretation of Clinical Practice Guideline for Low Back Pain Treated with Acupuncture and Moxibustion. Zhongguo Zhen Jiu 2015;35:1065-8.

28. Stürmer T, Raum E, Buchner M, et al. Pain and high sensitivity $\mathrm{C}$ reactive protein in patients with chronic low back pain and acute sciatic pain. Ann Rheum Dis 2005;64:921-5.

29. Poquet N, Lin C. The Brief Pain Inventory (BPI). J Physiother 2016;62:52.

30. Fairbank JC, Pynsent PB. The Oswestry Disability Index. Spine (Phila Pa 1976) 2000;25:2940-52; discussion 2952.

31. Carreon LY, Glassman SD, Howard J. Fusion and nonsurgical treatment for symptomatic lumbar degenerative disease: a systematic review of Oswestry Disability Index and MOS Short Form-36 outcomes. Spine J 2008;8:747-55.

32. Uher T, Bob P. Neuropathic pain, depressive symptoms, and C-reactive protein in sciatica patients. Int J Neurosci 2013;123:204-8.

33. Puyo CA, Peruzzi D, Earhart A, et al. Endotracheal tube-induced sore throat pain and inflammation is coupled to the release of mitochondrial DNA. Mol Pain 2017;13:1744806917731696.

34. Haghnegahdar A, Sedighi M. An Outcome Study of Anterior Cervical Discectomy and Fusion among Iranian Population. Neurosci J 2016;2016:4654109.
35. Li X, Han Y, Cui J, et al. Efficacy of Warm Needle Moxibustion on Lumbar Disc Herniation: A MetaAnalysis. J Evid Based Complementary Altern Med 2016;21:311-9.

36. Liu L, Sun XY, Lu Y, et al. Fire Needle Therapy for the Treatment of Psoriasis: A Quantitative Evidence Synthesis. J Altern Complement Med 2021;27:24-37.

37. Bai J, Han J, Zhu D, et al. Research and thinking on needling sensation of acupoint Huantiao (GB 30). Zhongguo Zhen Jiu 2015;35:253-6.

38. Wu JN, Liu ZS. Huantiao (GB 30): locating by muscle sulcus. Zhongguo Zhen Jiu 2012;32:752.

39. Qiu L, Hu XL, Zhao XY, et al. A Randomized Controlled Clinical Trial of Treatment of Lumbar Disc Herniationinduced Sciatica by Acupuncture Stimulation of Sciatic Nerve Trunk. Zhen Ci Yan Jiu 2016;41:447-50.

40. Jungen MJ, Ter Meulen BC, van Osch T, et al. Inflammatory biomarkers in patients with sciatica: a systematic review. BMC Musculoskelet Disord 2019;20:156.

41. Elkan P, Sten-Linder M, Hedlund R, et al. Markers of inflammation and fibrinolysis in relation to outcome after surgery for lumbar disc herniation. A prospective study on 177 patients. Eur Spine J 2016;25:186-91.

42. Lightowlers RN, Chinnery PF, Turnbull DM, et al. Mammalian mitochondrial genetics: heredity, heteroplasmy and disease. Trends Genet 1997;13:450-5.

43. Ryu C, Sun H, Gulati M, et al. Extracellular Mitochondrial DNA Is Generated by Fibroblasts and Predicts Death in Idiopathic Pulmonary Fibrosis. Am J Respir Crit Care Med 2017;196:1571-81.

44. Ashar FN, Zhang Y, Longchamps RJ, et al. Association of Mitochondrial DNA Copy Number With Cardiovascular Disease. JAMA Cardiol 2017;2:1247-55.

45. Nakahira K, Kyung SY, Rogers AJ, et al. Circulating mitochondrial DNA in patients in the ICU as a marker of mortality: derivation and validation. PLoS Med 2013;10:e1001577.

46. Zhan D, Tanavalee A, Tantavisut S, et al. Relationships between blood leukocyte mitochondrial DNA copy number and inflammatory cytokines in knee osteoarthritis. J Zhejiang Univ Sci B 2020;21:42-52.

Cite this article as: Hou WG, Pan HT, Wang RP, Feng CC, Pei LJ, Li J, Zhou J, Wang K. "Fan-zhen Jie-ci" acupuncture therapy for treatment of discogenic sciatica: protocol for a single-blind, randomized controlled clinical trial. Ann Palliat Med 2021;10(1):733-741. doi: 10.21037/apm-20-1418 


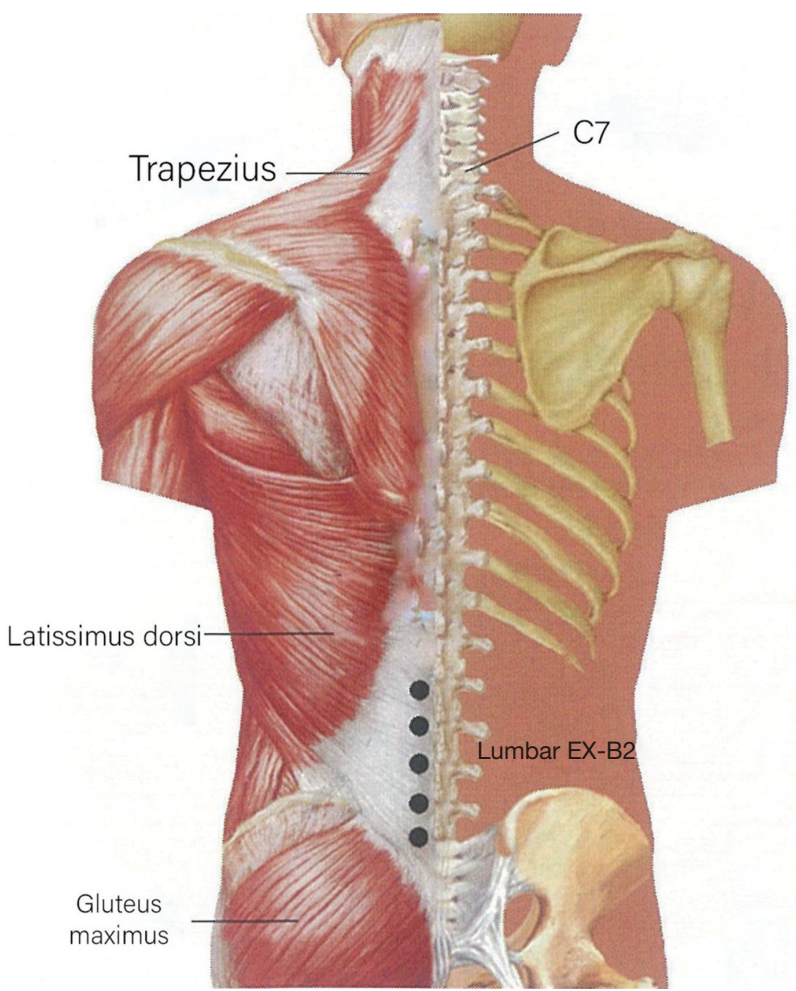

Figure S1 Location of lumber JiaJi (Ex-B2, unilateral). 